\title{
TEXTURE ANALYSIS FOR LAND USE LAND COVER (LULC) CLASSIFICATION IN PARTS OF AHMEDABAD, GUJARAT
}

\author{
Nizalapur Vyjayanthi ${ }^{*}$, Vyas Anjana \\ Centre for Applied Geomatics, CRDF, CEPT University, Navrangpura, Ahmedabad, Gujarat, India - 380009 \\ n.vyjayanthi@gmail.com, anjanavyas@yahoo.com
}

Commission III, WG III/2

KEY WORDS: SAR, RADARSAT-2, Grey Level Co-occurrence, Texture, Principal Component, Land Use Land Cover

\begin{abstract}
:
The present study addresses the potential of RADARSAT-2 data for Land Use Land Cover (LULC) Classification in parts of Ahmedabad, Gujarat, India. Texture measures of the original SAR data were obtained by the Gray Level Co-occurrence Matrix (GLCM). Results suggested False Colour Composite (FCC) of Mean, Homogeneity and Entropy showed a good discrimination of different land cover classes. Further, Principal Component Analysis (PCA) was also applied to the eight texture measures and FCC of Principal components is generated. Unsupervised classification is carried out for the above generated FCCs and accuracy assessment is carried out. The result of classification shows that the PCA generated from GLCM texture measures could obtain higher accuracy than using only the classification carried out by texture measures. Overall results of the study suggested possible use of single polarization and single date Radarsat-2 data for LULC classification with better accuracy using PCA generated image.
\end{abstract}

\section{INTRODUCTION}

Various studies showed that texture analysis in SAR data is the most important source of information (Ulaby et al. 1986, Dobson et al. 1986, Dell' Acqua et al. 2003, Luckman et al. 1997). Texture measures generated by grey level cooccurrence matrix (GLCM) has been explored in various studies (Marceau et al. 1990, Maillard, (2003) and Dulyakarn et al., 2000). Zakeri et al., 2017 stated that As texture features signify information regarding the spatial relation of pixel values, different features such as built-up urban areas, soil, rock, and vegetation, can be more accurately characterized.

Various studies proved that Grey Level Co-occurrence Matrix (GLCM) as the effective texture analysis scheme (Clausi et al. 2004; Kandaswamy et al. 2005). Haralick et al. (1973) proposed several measures that can be used to extract useful textural information from a GLCM. Many researchers used texture measures generated from GLCM matrix for land-cover mapping (Vander Sanden and Hoekman, 1999; Wu and Linders, 2000, Vyjayanthi, 2010). Gupta et al., 2014 analysed individual texture measures and decision tree classification technique was used for land cover classification.

Principal component analysis (PCA) is used to reduce dimensionality of a data set while retaining as much as possible the variation present in the data set and preserving discrimination ability. Various studies used Principal Component analysis for Land cover classification (Chamundeshwari et al, 2009, Zakeri et al., 2017).

Chamundeshwari et al., 2009 discussed the contribution of different texture measuresto improve the classification accuracy, It is found that the application of PCA transformation helps in integrating important information for classification purposes from all these feature measures.

The main objective of this study is to derive and analyse Grey Level Co-occurrence Measures (GLCM) derived texture measures for better depiction of LULC in study area. This paper is divided into three sections:

1. To find the combination of texture measures derived from Radarsat-2 HH polarization data for better and accurate depiction of LULC in study area. Unsupervised classification is performed and classified map of the study area is generated.

2. Principal Component Analysis is performed on generated texture measures and unsupervised classification is performed. Classified map of the study area is generated using this method.

3. Accuracy assessment is performed for the classified images using the ground data collected.

\section{STUDY AREA}

The study area is Ahmedabad city of Gujarat state. Ahmedabad is $5^{\text {th }}$ Largest city in terms of population and $7^{\text {th }}$ Largest Metropolitan city in India. Ahmedabad is an urban, densely populated industrialized largest city in the central part of the Gujarat state in Western India. Ahmedabad is divided by River Sabarmati into two physically distinct Eastern and Western regions. The location map of the study area is shown in Figure 1.

The land use/land cover (LULC) types were broadly divided into Urban (High Rise and Low Rise), Water (Rivers, Lakes,

\footnotetext{
* corresponding author
} 
and Canals), Vegetation (Crop land, Scrub, Parks), and Open land. The land cover in study area is diverse; certain land cover types exhibit similar scattering mechanisms, which make difficult to identify those features.

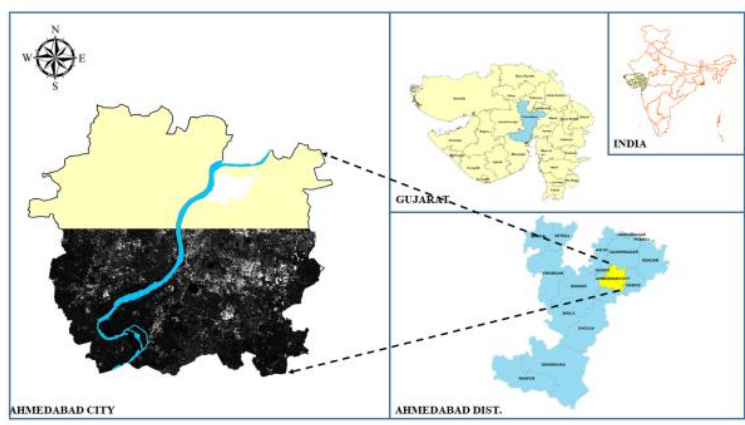

Figure 1. Location map of the study area and Radarsat-2 HH Polarization data used in present study

\section{DATASETS}

RADARSAT-2 images with fine quad-pol (FQ5) and Single Look Complex (SLC) obtained on $28^{\text {th }}$ September, 2017 was used in this study. The image has a full polarization of $\mathrm{HH}$, $\mathrm{HV}, \mathrm{VH}$, and $\mathrm{VV}$, and a resolution of $8.73 \mathrm{~m} \times 8.96 \mathrm{~m}$. The image is collected in descending mode with antenna positioning of right and an incidence angle of $32.98^{\circ}$.

\section{METHODOLOGY}

Radarsat-2 data of $\mathrm{HH}$ polarization acquired on 28 September, 2017 with incidence angle $33^{\circ}$ is considered in the present study. The study area is a metropolitan city comprising of High density and low density urban areas. Urban area mixed with vegetation can also be seen. Urban area with relatively nearby LULC classes viz., vegetation, wastelands and water bodies are delineated in the present study. The methodology followed is given in Figure 2. All processes in this section were done using Environment for Visualizing Images (ENVI) software (Exelis Visual Information, Boulder, CO, USA).

Pre-processing of the acquired data is carried out and GLCM texture measures were generated for $\mathrm{HH}$ polarized data with window size $5 \times 5$. The formulae of different texture measures are given below as discussed in Haralick et al., 1973 and also given in recent paper Zakeri et al., 2017.

1. Mean gives the mean value of the processing window.

$$
\text { Mean }=\sum_{i=2}^{2 N_{g}} i P_{x+y}(i)
$$

2. Variance is the local variance of the processing window and it is given by below equation.

Variance $=\sum_{i} \sum_{j}(i-\mu)^{2} P(i, j)$

3. Homogeneity is computed by the "inverse difference moment" equation given in equation 2 . Values range from 0 to 1.0

$$
\text { Homogeneity }=\sum_{i} \sum_{j} \frac{1}{1+(i-j)^{2}} P(i, j)
$$

$$
\begin{gathered}
\text { Contrast }=\sum_{n=0}^{N_{g}-1} n^{2}\left\{\sum_{i=1}^{N_{g}} \sum_{j=1}^{N_{g}} P(i, j)\right\} \\
|\mathrm{i}-\mathrm{j}|=\mathrm{n}
\end{gathered}
$$

5. Dissimilarity is computed by using the absolute values of the greyscale difference:

$$
\text { Dissimilarity }=\sum_{i, j=0}^{N_{g}-1} P_{i, j}\left(-\ln P_{i, j}\right)
$$

6. Entropy is computed by below equation.

$$
\text { Entropy }=-\sum_{i} P \log \left(P_{(i, j)}\right)
$$

7. Angular Second Moment (ASM) is computed from the following equation. It ranges from 0 to 1 .

$$
A S M=\sum_{i} \sum_{j}\left\{P_{(i, j)}\right\}^{2}
$$

8. Maximum probability shows the emergence of a pixel value adjacent to another pixel value more dominant in the image.

$$
\text { Maximum Probability }=\operatorname{Max} v\left(P_{(i, j)}\right)
$$

9. Correlation is computed using the following equation. Its values range from -1.0 to 1.0 .

$$
\text { Correlation }=\frac{\sum_{i} \sum_{j}(i j) P(i, j)-\mu_{x}-\mu_{y}}{\sigma_{x} \sigma_{y}}
$$

where $\mathrm{P}(\mathrm{i}, \mathrm{j})$ stands for $(\mathrm{i}, \mathrm{j})^{\text {th }}$ value in GLCM

$\mu_{\mathrm{x}}, \mu_{\mathrm{y}}, \sigma_{\mathrm{x}}$ and $\sigma_{\mathrm{y}}$ are the means and standard deviations of Px

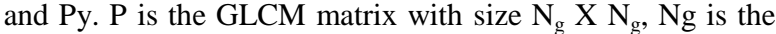
Number of gray levels

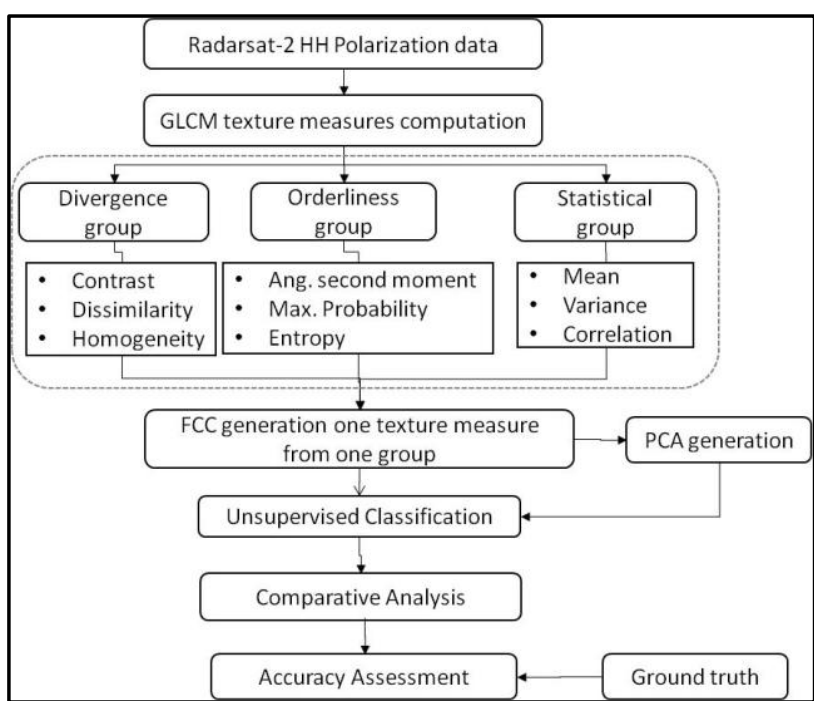

Figure 2. Methodology flow chart - Land Cover classification

4. Contrast is computed using the following equation: 
Usually, the texture measures generated are divided into three groups: 1) Contrast group (Contrast, Dissimilarity and Homogeneity); 2) Orderliness group (Angular second moment, Maximum Probability and Entropy); and 3) Statistical group (Mean, Variance and Correlation). One texture band from each group has been chosen and the better FCC combination is selected for further analysis. Principal Component Analysis is performed for the GLCM texture measures generated and the three principal components are selected based on the visualization of the better depiction of LULC in study area. PCA averages the pixels across the input images to compute a mean image. Optionally, it subtracts the computed image from each input image.

PCA transforms the original set of features to new axes where principal components corresponding to larger eigenvalues capture significant information represented by the original feature set. Three principal components are chosen from the result of PCA analysis. Unsupervised classification is performed for both the selected FCC combinations and accuracy assessment is carried out for the classified images using the ground truth data collected. Comparative analysis of the classified images are performed and discussed.

\section{RESULTS AND DISCUSSIONS}

Higher responses are observed for the densely built up areas as compared to the varying textures of medium gray levels for more vegetated built-up areas. Areas which has a mix of vegetation and built-up, respond in varying textures of medium gray, and individual buildings and building aggregates leads to individual higher responses. Each element in GLCM is related to a specific aspect of the texture such as directionality, distance, and correlation (Haralick et al. 1973).

As each texture measure gives different characteristics of land cover classes, these were used for interpretation and classification. Figure 3 shows the different texture measures generated for the study area.

In contrast group, Dissimilarity and contrast of the urban area are high whereas the Homogeneity of urban area is low. This may be attributed to the non-uniformity and occurrence of different scattering mechanisms in the urban area. Wide roads through the buildings or the varying units within the blocks contain both the single and double bounce scatterings. Low density urban area mixed with vegetation gives volume scattering from vegetations and double-bounce from buildings. This is also the reason why Homogeneity is less in mixed and low urban areas when compared to other classes. Homogeneity of the vegetation area and water bodies is high. High density urban areas have shown the high homogeneity values.

Considering the Orderliness group; Angular second moment, maximum probability of the urban area is low whereas Entropy of the urban area is high. This can be attributed to the dis-orderrness of the urban area. Maximum Probability and Angular second moment is high in wastelands and current fallow land as seen. Entropy of vegetation is low as compared to urban area. Waste lands and Water bodies have very low entropy values. In entropy band, there is clear distinction between urban, vegetation, waste lands and water bodies.

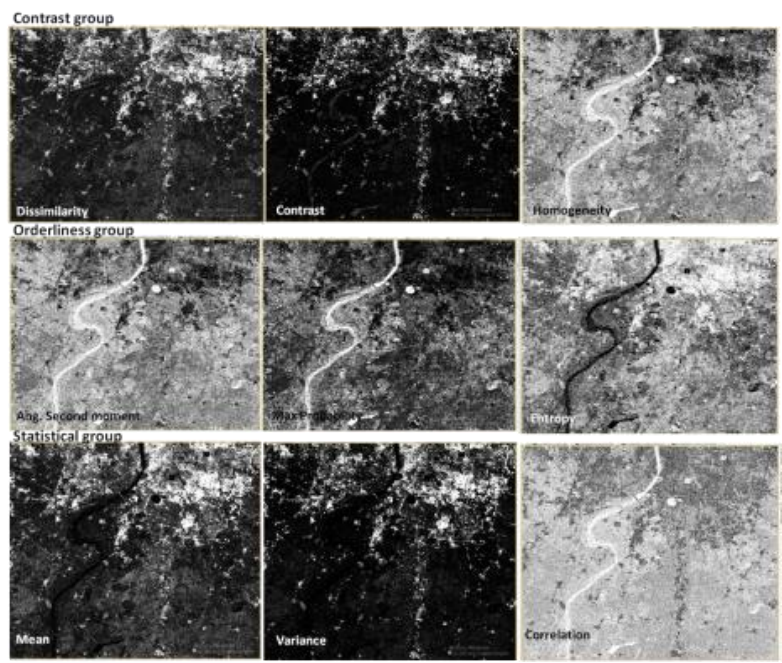

Figure 3. GLCM texture measures generated from Radarsat$2 \mathrm{HH}$ data

In statistical group; Mean and Variance of the urban areas is high as compared to correlation. As urban areas have higher backscatter values when compared to other LULC classes, the mean and variance are high in urban areas. This is followed by vegetation, waste lands and then water. The correlation value of water is high compared to other classes.

One band from each group is selected to include textural features of all three groups. In the present study, contrast band from Contrast group; Entropy band from Orderliness group and Mean band from Statistical group are selected and false colour composite (FCC) which is shown in Figure 3 is prepared for further analysis.

Urban areas have high backscatter compared to vegetation and bare soil. High backscatter values of urban areas are contributed by double bounce scattering mechanisms. As vegetation undergoes multiple scatterings and hence volume scattering, backscatter values of vegetation are lower than urban areas and higher than bare soil. Bare soil has low backscatter as it undergoes surface scattering. Backscatter values of water are low due to specular reflections. Roads and bridges can also be differentiated visually.

Figure 4 shows (a) Radarsat-2 HH polarization data, (b) FCC combination of chosen texture measures (Mean, Entropy and Homogeniety) and (c) Classified map derived from (b). In Figure 4 (a), clear distinction of urban area can be seen which is depicted in yellow colour. This is attributed to high mean backscatter, and high entropy values. Vegetation is seen in green colour due to medium mean and entropy values. There was no clear distinction between water and wastelands or non-vegetated areas. Here, backscatter values of water are low due to specular reflections and hence mean values are low. There was no clear distinction within the urban areas.

Unsupervised classification using ISODATA classifier is performed for figure 4 (b) and the classified map of the study area is shown in Figure 4(c). Confusion matrix, over all accuracy and kappa statistics are generated. Over all accuracy of the classified map is $65.96 \%$ and kappa statistics 0.56 . 
In fig 4 (b), though urban area is classified to some extent, there is misclassification of urban area with vegetation and bare soil. Dense urban area is classified accurately. Urban area mixed with vegetation is misclassified as vegetated area. Non-vegetated area is misclassified as water. Vegetated area is classified accurately.
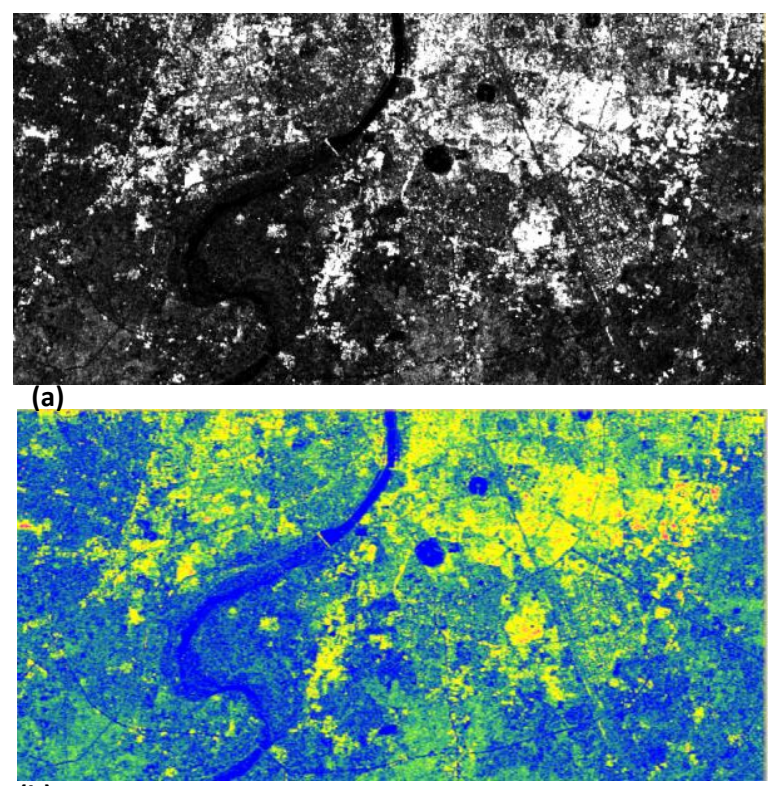

(b)

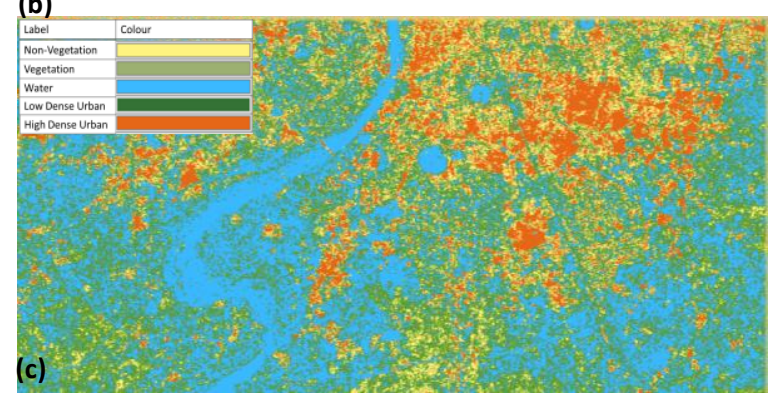

Figure 4. (a) HH polarization data (b) FCC of textures (Mean, Entropy and Homogeneity) generated from (a) and (c) Classified map of (b)

Principal Component Analysis was performed on the texture measures generated and FCC generated from Principal Components is shown in Figure 5 (a). Since PCA computes the correlation between input bands and sorts them based on the amount of data variance, the first components contain the greatest variance of the obtained texture measures (Zakeri et al., 2017).

PCA is used for dimensionality reduction and also to enhance classification accuracy for the single-band single polarized Radarsat-2 data. PCA fuses information from a number of various input features and gives output in terms of eigenvectors. They are orthogonal and capture all the information from the input features (Chamundeshwari et al., 2009).

Urban areas, Water, Vegetation and bare soil are visually discriminable in the image. Urban areas are depicted in yellow, red and magenta; Water and Non-vegetated areas are in blue; Vegetation in green.

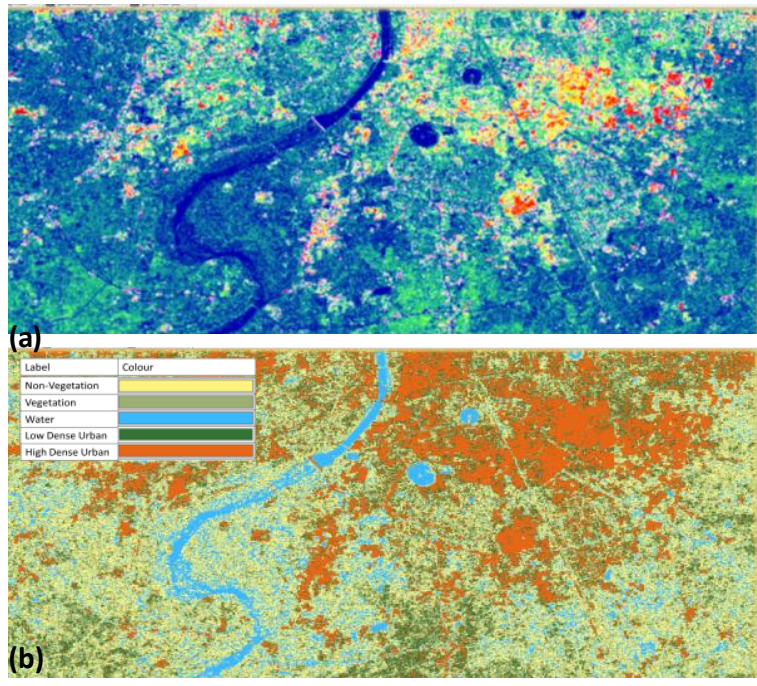

Figure 5. (a) FCC of PCs derived from GLCM textures and (b) Classified map of (a)

Unsupervised classification is performed on 5(a) and the classified map is shown in figure 5 (b). Confusion matrix, over all accuracy and kappa statistics are generated and given in Table 1. Over all accuracy of the classified map is $75.53 \%$ and kappa statistics 0.68. Urban areas are classified accurately. There is clear distinction between bare soil and water unlike figure 4 (b). Classification accuracy with urban areas and different land cover classes has improved when compared to earlier one.

\begin{tabular}{|l||c||c||c||c||c||c||c|}
\hline \multicolumn{1}{|c|}{ Class } & $\begin{array}{l}\text { Open } \\
\text { land }\end{array}$ & Vegetation & Water & $\begin{array}{l}\text { low dense } \\
\text { urban }\end{array}$ & $\begin{array}{l}\text { High dense } \\
\text { urban }\end{array}$ & Total & $\begin{array}{c}\text { Producer's } \\
\text { Accuracy (\%) }\end{array}$ \\
\hline \hline Open land & 45 & 9 & 0 & 2 & 2 & 58 & 77.59 \\
\hline Vegetation & 2 & 42 & 0 & 1 & 1 & 46 & 91.30 \\
\hline Water & 13 & 2 & 14 & 0 & 2 & 31 & 45.16 \\
\hline Low dense urban & 1 & 2 & 0 & 24 & 2 & 29 & 82.76 \\
\hline High dense urban & 1 & 3 & 0 & 3 & 17 & 24 & 70.83 \\
\hline Total & 62 & 58 & 14 & 30 & 24 & 188 & 73.53 \\
\hline \hline $\begin{array}{c}\text { User's accuracy } \\
\text { (\%) }\end{array}$ & 72.58 & 72.41 & 100.00 & 80.00 & 70.83 & 79.17 & \\
\hline \hline
\end{tabular}

Table 1. Confusion matrix of classified map generated from PCA generated from GLCM texture measures

\section{CONCLUSIONS}

In the present study, Land use land Cover Classification in parts of Ahmedabad city, Gujarat, India is carried out by stand alone Radarsat-2 HH polarization data.. GLCM texture measures were generated from RADARSAT-2 data and classification is carried out on FCC generated from Mean, Entropy and Homogeneity. Further, PCA is applied on the generated texture measures and classification is performed on FCC of principical components. It is found that the application of PCA to the generated texture measures has increased the classification accuracy and also delineation of different classes is accurate.

Single date single polarization data for land cover classification can be improved by performing Principal Component Analysis on generated GLCM texture measures. 


\section{ACKNOWLEDGEMENTS}

The authors gratefully acknowledge Department of Science and Technology (DST), New Delhi, Government of India for the financial support under Women Scientist programme, WOS-A Scheme; File No: SR/WOS-A/EA-17/2016 (G).

\section{REFERENCES}

Chamundeeswari, V.V., D. Singh and K. Singh. 2009. "An Analysis of Texture Measures in PCA-Based Unsupervised Classification of SAR Images." IEEE Trans. Geosci. Remote Sens. Letters, 6(2), 214-218.

Clausi, D.A.; Yu, B. Comparing co-occurrence probabilities and Markov random fields for texture analysis of SAR Sea ice imagery. IEEE Trans. Geosci. Remote Sens. 2004, 42, 215-228.

Dell Acqua, F., and Gamba, P., 2003, Pyramidal rain field decomposition using radial basis function neural networks for tracking and forecasting purposes. IEEE Trans. Geosci. Remote Sens. 41(4), 853.

Dobson, M.C., and Ulaby, F.T., 1986, Active microwave soil moisture research. IEEE Trans. Geosci. Remote Sens. 24(1), 23-36.

Dulyakarn, P., Rangasanseri, Y., and Thitimajshima, P., 2000, Comparison of two features for multispectral imagery analysis. Proceeding of Asian Conference of Remote Sensing.

S. Gupta, D. Singh and S. Kumar, "An approach based on texture measures to classify the fully polarimetric SAR image," 2014 9th International Conference on Industrial and Information Systems (ICIIS), Gwalior, 2014, pp. 1-6, doi: 10.1109/ICIINFS.2014.7036651.

Haralick, R.M., Shanmugam, K., and Dinstein, I., 1973, Textural features for image classification, IEEE Trans. Syst., Man, Cybern. Syst. 3, 610-621.
Homa Zakeri, Fumio Yamazaki and Wen Liu, 2017. Texture Analysis and Land Cover Classification of Tehran Using Polarimetric Synthetic Aperture Radar Imagery. Appl. Sci.. 7, 452.

Kandaswamy, U.; Adjeroh, D.A.; Lee, M.C. 2005 Efficient Texture Analysis of SAR Imagery. IEEE Trans. Geosci. Remote Sens. 43, 2075-2083.

Luckman, A.J., Kuplich, T.M., Yanasse, C.C.F., and Frery, A.C., 1997, A study of the relationship between radar backscatter and regenerating forest biomass for spaceborne SAR instruments. Remote Sens. Environ. 60, 1-13.

Maillard, P., 2003, Comparing texture analysis methods through classification. Photogramm Eng Remote Sens 69(4), 357-367.

Marceau, D.J., Howarth, P.J., Dubois, J.M., Gratton, D.J., 1990, Evaluation of the grey-level co-occurrence matrix method for land-cover classification using SPOT imagery. IEEE Trans. Geosci. Remote Sens. 28, 513-519.

Ulaby, F.T., Moore, R.K., \& Fung, A.K., 1986, (Debham, Massachusetts: Artech House, Inc). Microwave Remote Sensing, Active and Passive. Volume Scattering and Emission Theory- Advanced Systems and Applications.

Van der Sanden, J.J. and D.H. Hoekman, 1999, Potential of airborne radar to support the assessment of land cover in a tropical rain forest environment, Remote Sens. Environ. 68, 26-40.

Vyjayanthi, N, 2010, Synthetic Aperture radar data analysis for vegetation classification and biomass estimation of tropical forest area, Ph.D. Thesis, JNTU, Hyderabad, India.

Wu, D., Linders J., 2000. Comparison of three different methods to select feature for discriminating forest cover types using SAR imagery. Int J Remote Sens. 21, 20892099. 